about 800 chronic psychiatric patients in hospital, mostly schizophrenic. About 200 of them are acutely psychotic and those in remissions also need prolonged pharmacologic treatment.

We currently lack most antipsychotic drugs, some not available now in Croatia, and others too expensive for our limited funds. So, we are short of neuroleptic drugs, antiparkinsonians and benzodiazepines.

In better times we mostly used haloperidol, flufenazine, clozapine, promazine, chlorpromazine, thioridazin, carbamazepine, levamepromazine, buperidine, triheksifenidil, diazepam, nitrazepam, flurazepam, lorazepam.

If you are able to help us in any way we would appreciate it very much.

We hope to hear from you soon.

SANJa Martić-Biočrina

JOSKKO VULETIN

\author{
Neuropsychiatric Hospital \\ "Dr Ivan Barbot" \\ 41317 Popovaca \\ Croatia
}

\section{The reliability of delivery of interim discharge slips by psychiatric patients DEAR SIRS}

Kerr (1990) expressed doubts about the reliability of the psychiatric patient as a messenger for communication between the hospital and the family doctor. A comprehensive study has been carried out on medical patients comparing the reliability and speed of arrival of interim discharge slips sent by hand and by post (Sandler \& Mitchell, 1987). They found that $97 \%$ of the slips in both groups arrived, and that $55 \%$ of the by hand group arrived within one day. No such work has been carried out on psychiatric patients.

I took 50 consecutive discharges from an acute admission ward. Each acted as his or her own control having a by hand and a by post slip. The slip contained information as to the nature of the admission, follow-up arrangements and the medication on discharge. The doctors' surgeries were contacted by telephone to determine arrival of the slips.

Ninety-eight per cent of the slips sent by post arrived compared with $66 \%$ of those sent by hand. Of the 32 pairs, where both arrived, a Wilcoxon Rank Sign test was statistically significant at $P<0.01$. The by post group had a median delivery time of four days compared with one day for the by hand group. There was no significant difference between diagnostic groupings or when Caucasians were compared with Asians.

The posted interim slips therefore arrived more reliably but slower compared with those sent by hand. As the quantity of tablets for a patient to take home gets smaller and the cost of postage rises, I felt it was important to know the reliability of delivery of discharge slips in psychiatric patients. The reluctance to tell the general practitioner of the psychiatric admission may be due to the perceived stigma of mental illness or to lack of insight as part of the illness.

Ideally each patient should have both a by hand and a by post slip (the former ensuring that the latter is written before the patient leaves the hospital). A triplicated pad would allow a record also to be kept in the notes. Where there is only one interim slip this should be posted to ensure reliable delivery. This may lead to practice being changed in some hospitals.

ANN K. Stanley

\section{Reaside Clinic}

Bristol Road South, Birmingham B45

\section{References}

KERR, M. S. D. (1990) Psychiatric discharge summaries. British Medical Journal, 300, 260-261.

SANDler, D. A. \& Mitchell, J. R. A. (1987) Interim discharge summaries how are they best delivered to general practitioners? British Medical Joumal, 295, 1523-1525.

\section{Junior doctor representation}

\section{DeAR Sirs}

At the end of the article 'Working for Trainees' (Psychiatric Bulletin, February 1993, 17, 98-99), the authors' state that they are aware of at least one other active organised junior psychiatric trainee group working at a regional level. Such a group has been set up in South West Thames, with very active trainee participation.

The St George's professional scheme comprises four registrar rotations and two central SHO rotations, which together account for approximately 90 trainees. Each hospital on the scheme has its own local representative, and each of the SHO or registrar rotations has an elected representative. There is a chairman who is elected by all members of the rotation, and represents trainees' views at regional level.

The corner-stone of representation is the twomonthly junior doctors' meeting which is attended by all representatives. St George's Hospital also has a local BMA representative, two trainee members of the Royal College Training Committee and a senior registrar representative. These meetings are a means of conveying problems at local level so that they may be discussed and a possible strategy reached. This organisation has been responsible in part for improving the quality of some of the less desirable jobs on each rotation, and as in Liverpool the trainee criticism seems to have been viewed as constructive by the psychiatric tutors. 